\title{
Few Mode Ring-Core Fibre Amplifier for Low Differential Modal Gain
}

\author{
Y. Jung, ${ }^{1,{ }^{*}}$ Q. Kang, ${ }^{1}$ L. Shen, ${ }^{2}$ S. Chen, ${ }^{2}$ H. Wang,${ }^{2}$ Y. Yang, ${ }^{2}$ K. Shi, ${ }^{3}$ B. C. Thomsen,${ }^{3}$ \\ R. Amezcua Correa, ${ }^{4}$ Z. Sanjabi Eznaveh, ${ }^{4}$ J. Carlos Alvarado Zacarias, ${ }^{4}$ J. Antonio-Lopez, ${ }^{4}$ \\ P. Barua, ${ }^{1}$ J. K. Sahu, ${ }^{1}$ S. U. Alam, ${ }^{1}$ and D. J. Richardson ${ }^{1}$
}

\begin{abstract}
${ }^{1}$ Optoelectronics Research Centre, University of Southampton, SO17 1BJ, United Kingdom ${ }^{2}$ State Key Laboratory of Optical Fiber and Cable Manufacture Technology, Yangtze Optical Fiber and Cable Joint Stock Limited Company, Wuhan, China

${ }^{3}$ Department of Electronics and Electrical Engineering, University College London, London, WC1E 7JE, United Kingdom

${ }^{4}$ CREOL, the College and Optics and Photonics, the University of Central Florida, Orlando, Florida 32816, USA

*Corresponding Authors: ymj@orc.soton.ac.uk
\end{abstract}

\begin{abstract}
We report a few-mode ring-core fibre amplifier for mode division multiplexed transmission. We achieve very low differential modal gain $(<1 d B)$ for 5 spatial modes over the $C$-band highlighting the benefits of the ring core approach.
\end{abstract}

\section{Introduction}

Few mode erbium-doped fibre amplifiers (FMEDFAs) are one of the key building blocks required for the successful implementation of mode division multiplexed (MDM) transmission systems [1, 2] and various active fibre designs have [3-5] been proposed to achieve low differential modal gain (DMG). In a step-index, uniformly doped FM-EDFA the management of DMG becomes more and more challenging as the number of spatial modes is increased since the intensity profiles of the signal mode differ widely from each other. Complicated erbium doping profiles in conjunction with selective pump mode control are thus generally required. In comparison, few-mode ring-core EDFAs (FMRC-EDFAs) offer inherent advantages in terms of reducing DMG because the intensity profiles of the ring modes are all very similar. This means that they each experience similar gain/absorption in a uniformly doped FM-RC-EDF thereby removing the need for highly sophisticated doping profiles. Recently we have theoretically proposed a FM-RC-EDFA capable of providing almost identical gain among six mode groups within the C-band using either core- or claddingpumped implementations [6]. In reference [7], the first ring-core fibre (RCF) amplifier supporting two mode groups $\left(\mathrm{LP}_{01}\right.$ and $\left.\mathrm{LP}_{11}\right)$ has been demonstrated and a small DMG of around $1 \mathrm{~dB}$ was realized.

In this paper, we report a five mode ring-core erbium doped fibre amplifier (5M-RC-EDFA) with very low DMG $(<1 \mathrm{~dB})$. This experimental demonstration proves again the advantages of the ring-core approach for the realization of low DMG amplifiers.

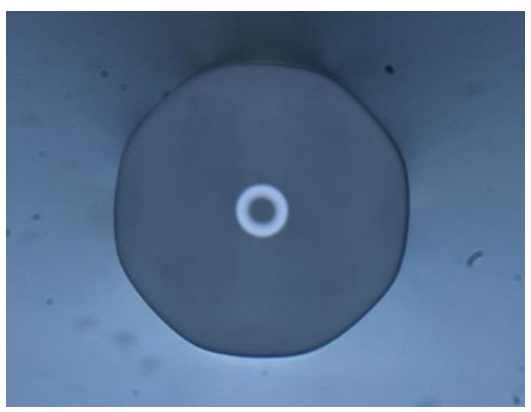

(a)

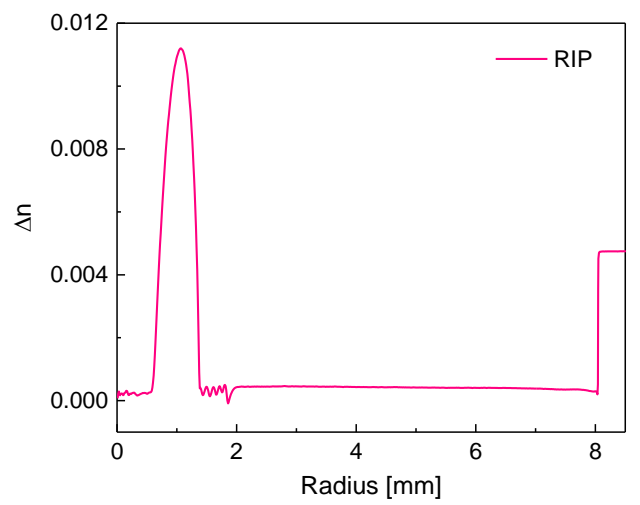

(b)

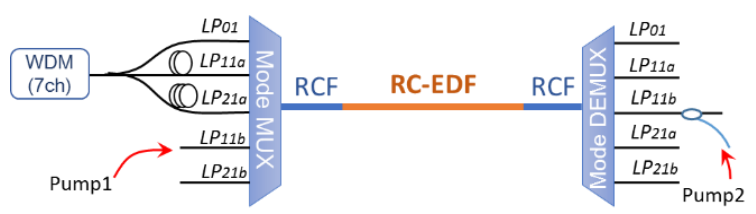

(c)

Fig. 1: (a) Cross-sectional microscope image and (b) refractive index profile of the fabricated 5M-RC-EDF and (c) the measurement setup for modal gain analysis of the amplifier. 


\section{Fabrication and characterization of 5M-RC- EDFA}

Our Er-doped RCF was designed to support 5 spatial modes with a single-radial-order (i.e. $\mathrm{LP}_{01}, \mathrm{LP}_{11 \mathrm{a}}, \mathrm{LP}_{11 \mathrm{~b}}, \mathrm{LP}_{21 \mathrm{a}}$ and $\left.\mathrm{LP}_{21 \mathrm{~b}}\right)$. It was fabricated using a conventional modified chemical vapor deposition (MCVD) process in conjunction with solution doping. The $110 \mu \mathrm{m}$ outer glass cladding was octagonally-shaped to enable cladding-pumping. The preform refractive index profile and cross-sectional microscope image of the fabricated $5 \mathrm{M}-\mathrm{RC}$ EDF are shown in Fig. 1(a,b). The fibre core can be clearly seen as a bright annular ring. The inner radius of the annular ring is $5.8 \mu \mathrm{m}$ while the outer radius is $9.4 \mu \mathrm{m}$. The core-to-clad refractive index difference is $\Delta n=0.0112$ corresponding to an NA of 0.18 . Figure 1(c) shows a schematic of the 5M-RC-EDFA and the associated amplifier test setup. Seven WDM signals were split into three optical paths, each was mode-multiplexed by a mode-selective photonic lantern $(\mathrm{PL})$ and launched into a length of matched passive RCF [8]. The photonic lanterns were in-house fabricated [9] with a mode dependent insertion loss of about 1.8-3 $\mathrm{dB}$ and an extinction ratio of $>8 \mathrm{~dB}$. The passive RCF tail of the PL was directly spliced to one end of the active RC-EDF while the other end of the RC-EDF was spliced to a second PL for mode-demultiplexing. The mode dependent splice losses between the active and passive RCFs were 1.8, 2.0 and $3.4 \mathrm{~dB}$ for $\mathrm{LP}_{01}, \mathrm{LP}_{11}$ and $\mathrm{LP}_{21}$, respectively. Originally, the active fibre was intended for cladding-pumping however the fabricated fibre exhibited high background loss at $1550 \mathrm{~nm}$ (more than a few $\mathrm{dB} / \mathrm{m})$, which made it difficult to achieve reasonable gain with the few meters long active fibre required given the peak cladding pump absorption of $2.4 \mathrm{~dB} / \mathrm{m}$ at $979 \mathrm{~nm}$. A core pumped configuration was therefore employed instead to reduce the active fibre length. A 27 $\mathrm{cm}$-long 5M-RC-EDF was used in our experiment. As shown in Fig. 1(c), two singlemode pump laser diodes $(27.5 \mathrm{dBm}$ each) were coupled in a bi-directional pump configuration (one coupled via the $\mathrm{LP}_{11 b}$ port of the input $\mathrm{PL}$ and the other similarly coupled through the $\mathrm{LP}_{11 b}$ port of the output $\mathrm{PL}$ ). The total coupled pump power was estimated to be $\sim 23 \mathrm{dBm}$ due to the insertion loss of the PL and splice loss between the passive and active fibres.

First, we examined both the near- and far-field distributions of the amplified spatial modes by pumping through the input $\mathrm{PL}$ and without splicing the output PL. Clean modal amplification was confirmed at the output end of the $5 \mathrm{M}$-RC-EDF using a CCD camera as shown
(Far field mode profiles)
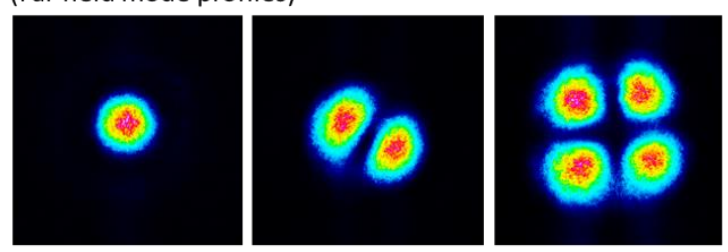

(Near field mode profiles)
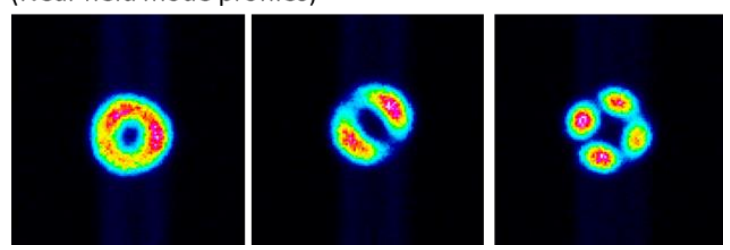

(a)

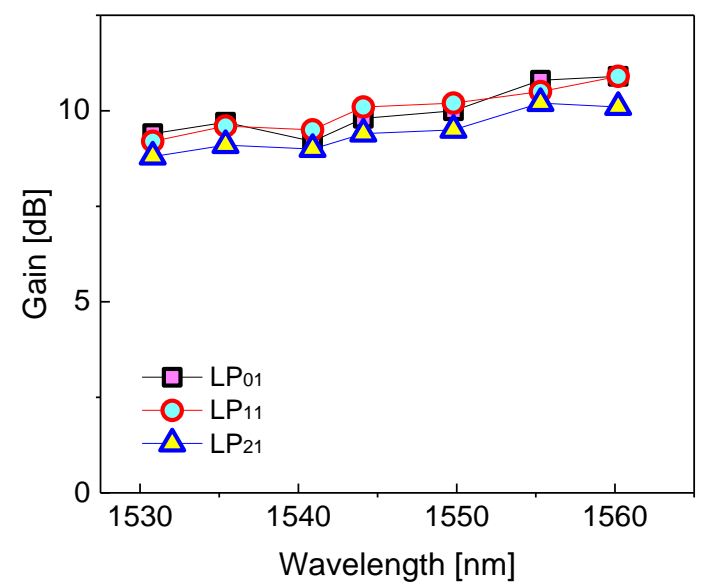

(b)

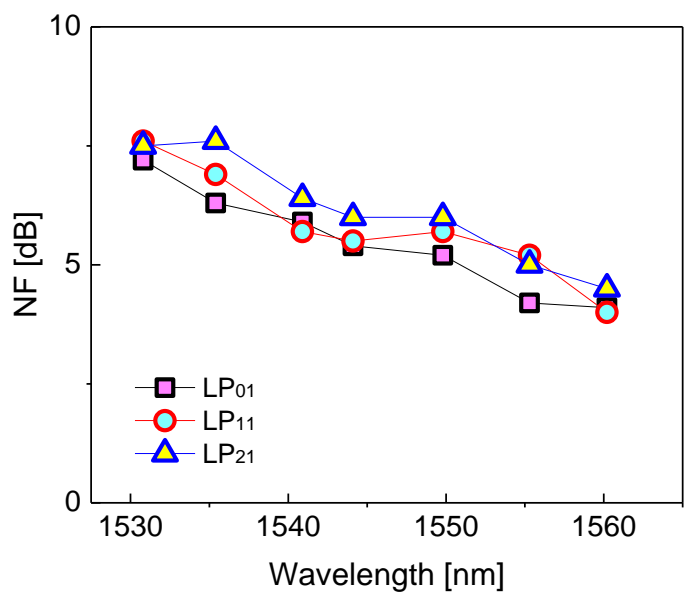

(c)

Fig. 2: (a) Far- and near-fields of the amplified signal modes and (b) measured modal gain and (c) noise figure as a function of wavelength at a fixed input signal power of

$-15 \mathrm{dBm}$ and launched pump power of $23 \mathrm{dBm}$.

in Fig. 2(a). The detailed amplifier performance was then analyzed using the output $\mathrm{PL}$ as a mode demultiplexer. Fig. 2(b, c) shows the measured modal gain and noise figure (NF) as a function of wavelength at a fixed input signal power of $-15 \mathrm{dBm}$ per mode and a total launched pump power of $23 \mathrm{dBm}$. The average modal gain was relatively low $(\sim 10 \mathrm{~dB})$ due 
primarily to the relatively low launched pump power and the short fibre length used to minimize the impact of fibre background loss. The increase in gain with wavelength signifies the lack of population inversion in the RC-EDF. Consequently the NF of the amplifier as plotted in Fig. 2(b) also shows an upward trend at the short wavelength edge reaching to $7.5 \mathrm{~dB}$ at $1530 \mathrm{~nm}$. However, a very low DMG (less than $1 \mathrm{~dB}$ ) was successfully achieved in the wavelength range of $1530-1560 \mathrm{~nm}$. Note that this is the lowest experimentally measured DMG achieved so far from a FM-EDFA. The primary reason for the large observed RCF loss is associated with the relatively low index contrast between the core and cladding in this first generation of fabricated fibre which compromises the guidance strength (or core confinement) under single radial mode guidance conditions. We strongly believe that with realistic improvements in the index contrast that high performance FM-EDFAs (e.g. average gain $>20 \mathrm{~dB}, \mathrm{DMG}<1 \mathrm{~dB}$ ) will be achievable with the proposed ring-core fibre amplifier.

\section{Conclusions}

We have experimentally demonstrated a 5 spatial mode ring-core fibre amplifier for mode division multiplexed transmission. A very low differential modal gain of less than $1 \mathrm{~dB}$ over the C-band with an average modal gain of $\sim 10 \mathrm{~dB}$ was successfully achieved.

\section{Acknowledgements}

This work was supported by the UK EPSRC grant EP/J008591/1 (COMIMO) and the Hubei, China 2015CFB705 funding.

\section{References}

[1] D. J. Richardson et al., "Space division multiplexing in optical fibres", Nat. Photonics 7, p. 354 (2013).

[2] V. Sleiffer et al., "73.7 Tb/s $(96 \times 3 \times 256 \mathrm{~Gb} / \mathrm{s})$ mode division multiplexed DP-16QAM transmission with inline MM-EDFA," Opt. Express 20, B428 (2012).

[3] Y. Jung et al., "First demonstration and detailed characterization of a multimode amplifier for space division multiplexed transmission system," Opt. Express 19, B.952 (2011).

[4] Y. Jung et al., "three mode Er3+ ring-doped fiber amplifier for mode-division multiplexed transmission," Opt. Express 21, p. 10383 (2013).

[5] Y. Jung et al., "Cladding pumped few-mode EDFA for mode division multiplexed transmission," Opt. Express 22, p. 29008 (2014).

[6] Q. Kang et al., "Gain equalization of a six-mode group ring core multimode EDFA" Proc. ECOC, P.1.14, Cannes (2014).

[7] H. Ono et al., "2-LP mode few-mode fiber amplifier employing ring-core erbium doped fiber," Opt. Express 23, p. 27405 (2015).

[8] Y. Jung et al., "Low-loss $25.3 \mathrm{~km}$ few-mode ring-core fiber for mode division multiplexed transmission," J. Lightwave Technol. 35, p. 1363 (2017).
[9] Z. S. Eznaveh et al., "Annular core photonic lantern spatial mode multiplexer," Proc. OFC, Tu3J.3, Los Angeles (2017). 